developed ELISA-based RF assays. RF responses were analysed in healthy donors, arthralgia patients at risk for RA and RA patients at different disease stages.

Results: RF responses in all cohorts were shown to be primarily directed against epitopes at the interface of the second and third $\lg$ Geavy chain domains $(\mathrm{CH} 2$ $\mathrm{CH} 3$ ) and the tip of the $\mathrm{CH} 3$ domain. The generated target antibodies were able to separate these RF responses and discriminate them from RF responses targeting epitopes outside these reactivity hotspots. Furthermore, certain RF responses against the $\mathrm{CH} 2-\mathrm{CH} 3$ interface were shown to depend on the presence of a single specific amino acid residue.

Pilot experiments using the target antibodies in clinical cohorts revealed that, in arthralgia patients and RA patients at various disease stages, the pattern of RF reactivity differs between individual patients as well as between cohorts. The degree to which an RF response against the $\mathrm{CH} 2-\mathrm{CH} 3$ interface depends on the single specific amino acid residue varied substantially between patients. One particular RF response pattern, RFs binding only the $\mathrm{CH} 3$ domain, was found exclusively in non-RA patients and healthy donors.

Conclusions: Using newly developed target lgGs, RF responses against different epitopes can be characterised. RF response patterns differ between RA and non-RA cohorts and between RA patients. These new tools may lead to identification of RA-specific RF responses and provide more insight into the pathogenic role of these autoantibodies.

Disclosure of Interest: None declared

DOI: 10.1136/annrheumdis-2018-eular.3079

\section{SAT0018 BLOOD CHEMOKINE SYSTEM PROFILE ASSOCIATED WITH DISEASE ACTIVITY IN RHEUMATOID ARTHRITIS}

A. Petrackova ${ }^{1}$, G. Gabcova ${ }^{1}$, M. Skacelova ${ }^{2}$, Z. Mikulkova ${ }^{1}$, A. Smrzova ${ }^{2}$, M. Schubertova ${ }^{2}$, F. Mrazek ${ }^{1}$, P. Horak ${ }^{2}$, E. Kriegova ${ }^{1} .{ }^{1}$ Department of Immunology; ${ }^{2}$ Department of Internal Medicine III - Nephrology, Rheumatology and Endocrinology, Faculty of Medicine and Dentistry, Palacky University Olomouc, Olomouc, Czech Republic

Background: Chemokine receptors and their chemokine ligands (chemokine system), key mediators of inflammatory and immune cell trafficking, are involved in the pathogenesis of rheumatoid arthritis (RA). There is still limited information of blood chemokine system signature associated with disease activity.

Objectives: To identify RA-associated chemokine system signature using highly sensitive multiplex Proximity Extension ImmunoAssay (PEA) in serum together with analysis of CCR5, CCR6 and CXCR3 expression in blood cell subsets and assess its relationship with disease activity as evaluated by activity score (DAS28).

Methods: We investigated the serum levels of 92 inflammation-related proteins in 78 Czech patients with RA by PEA (Proseek Multiplex, Olink Bioscience, Sweden). Disease activity was assessed by means of DAS28 and subgroups were formed based on the disease activity, where DAS28 of $>3.2$ was taken as active $R A$ (inactive $R A, n=40$; active $R A, n=38$ ). The expression of CCR5, CCR6 and CXCR3 receptors were analysed using 6-colour flow cytometry (BD FACSCanto II) on T and B lymphocytes, NK, dendritic cells, and monocytes in peripheral blood from 11 patients with active RA and 9 age-matched healthy control subjects. Statistical tests (Mann-Whitney $U$ test, Spearman correlation) were performed using GenEx (Sweden) and GraphPad Prism 5.01 (CA, USA). P-value $\leq 0.05$ was considered as significant.

Results: In patients with active RA, 16 serum proteins were upregulated $\left(P_{\text {corr }} \leq 0.05\right)$. Top-ranked proteins distinguishing active and inactive RA were CCL20, CXCL9, CCL7, CXCL10, IL-6, sCDCP1, and CXCL1 ( $\left.P_{\text {corr }}<0.03\right)$. Levels of all these proteins positively correlated with disease activity $(r>0.30, p<0.006)$ and the best correlation was observed for chemokines CCL20 ( $r=0.495$, $p=0.000004)$ and CCL7 $(r=0.442, p=0.00005)$. Based on these results we analysed the expression of corresponding chemokine receptors: CCR5, CCR6, and CXCR3 in blood cells. Expression of CCR6 was increased on CD4 $+\mathrm{T}$ and CD8 $+\mathrm{T}$ lymphocytes $(\mathrm{p}=0.008, \mathrm{p}=0.01$ respectively) and on total and activated NK cells ( $p=0.01, p=0.004$ respectively) in active RA compared with healthy controls. Regarding CCR5, decreased percentage of CCR5 positive CD4 + T lymphocytes were registered in RA patients compared with healthy controls $(p=0.008)$. No differences were observed in the expression of CXCR3 on investigated immune cell populations.

Conclusions: We identified the blood chemokine system signature associated with disease activity in RA further supporting a critical role of CCL20/CCR6 axis in the ongoing inflammation related to the disease activity in RA.

Acknowledgements: Grant support: MZ CR VES15-28659A,

IGA LFUP 201816

Disclosure of Interest: None declared

DOI: 10.1136/annrheumdis-2018-eular.6523

\section{SAT0019 \\ TNF-A REGULATES PLASMACYTOID DENDRITIC CELLS BY SUPPRESSING IFN-A PRODUCTION AND} ENHANCING TH1 AND TH17 CELL DIFFERENTIATION

A. Psarras ${ }^{1,2}$, A. Antanaviciute ${ }^{3}$, I. Carr ${ }^{3}$, M. Wittmann ${ }^{1}$, P. Emery ${ }^{1}$, G. Tsokos ${ }^{2}$, E. M. Vital ${ }^{1} .{ }^{1}$ Leeds Institute of Rheumatic and Musculoskeletal Medicine, University of Leeds, Leeds, UK; ${ }^{2}$ Beth Israel Deaconess Medical Center, Harvard Medical School, Boston, USA; ${ }^{3}$ Leeds Institute for Data Analytics, University of Leeds, Leeds, UK

Background: Human plasmacytoid dendritic cells ( $\mathrm{pDCs}$ ) play a vital role in modulating immune responses. pDCs can produce massive amounts of type I IFNs in response to nucleic acids via toll-like receptors (TLRs) and they are known to possess weak antigen-presenting properties inducing $\mathrm{CD}^{+} \mathrm{T}$ cell activation. Previous data showed a cross-regulation between TNF- $\alpha$ and IFN- $\alpha$ but the effect of TNF- $\alpha$ on $p D C$ s remains unclear.

Objectives: To investigate how TNF- $\alpha$ regulates the immune function of human pDCs.

Methods: Fresh PBMCs were treated with TNF- $\alpha$, TLR7 and TLR9 synthetic agonists. pDCs were immunophenotyped using flow cytometry. RNA from sorted pDCs was extracted and sequenced using Smart-seq2 for sensitive full-length transcriptome profiling. For $\mathrm{pDC} / \mathrm{T}$ cell co-culture, fresh or TNF- $\alpha$-treated $\mathrm{pDCs}$ were cultured with naîve $C D 4^{+} T$ cells for 5 days. The production of cytokines was measured by intracellular staining and ELISA.

Results: Upon stimulation with TLR7 and TLR9 agonists, there were three main pDC populations: non-producers, TNF- $\alpha$-producers, TNF- $\alpha / \mathrm{IFN}-\alpha$-producers. Exogenous TNF- $\alpha$ significantly reduced the production of both IFN- $\alpha$ and TNF- $\alpha$ in TLR9-stimulated pDCs but only IFN- $\alpha$ in TLR7-stimulated pDCs. Neutralisation of autologous TNF- $\alpha$ with anti-TNF antibody partially sustained IFN- $\alpha$ secretion by TLR9-stimulated pDCs after 24 hours. Exogenous TNF- $\alpha$ significantly promoted $\mathrm{pDC}$ maturation by upregulation of costimulatory molecules and chemokine receptors such as CD80, CD86, HLA-DR, and CCR7. RNAseq data analysis suggested that TNF- $\alpha$ inhibits IFN- $\alpha / \mathrm{TNF}-\alpha$ production by interfering with the NFKB pathway, promotes antigen processing and presentation pathways as well as T cell activation and differentiation. Indeed, TNF- $\alpha$-treated pDCs induced in vitro higher $\mathrm{CD} 4^{+} \mathrm{T}$ cell proliferation and favoured Th1/Th17 polarisation.

Conclusions: Although pDCs possess weak antigen-presenting properties, TNF- $\alpha$ can enhance pDC maturation by switching their main role as IFN- $\alpha$-producing cells to a more conventional DC phenotype. The functional status of pDCs might be strongly influenced by overall inflammatory environment and TNF- $\alpha$ might regulate IFN- $\alpha$-mediated aspects of a range of autoimmune and inflammatory diseases.

Disclosure of Interest: None declared

DOI: 10.1136/annrheumdis-2018-eular.4688

\section{SAT0020 IMPACT OF IL12B SINGLE NUCLEOTIDE POLYMORPHISMS ON CIRCULATING PRO- INFLAMMATORY CYTOKINES IL-12P40 AND IL-23 IN ANKYLOSING SPONDYLITIS}

M.G. Ivanova ${ }^{1}$, I. Manolova ${ }^{2}$, R. Stoilov ${ }^{1}$, L. Miteva ${ }^{2}$, S. Stanilova ${ }^{2} .{ }^{1}$ Clinic of Rheumatology, University Hospital "St. Iv. Rilski", Medical Faculty, Medical University, Sofia; ${ }^{2}$ Department of Molecular Biology, Immunology and Medical Genetics, Medical Faculty, Trakia University, Stara Zagora, Bulgaria

Background: Ankylosing spondylitis (AS) is an immune-mediated rheumatic disease belonging to the spectrum of axial spondyloarthritides, characterised mainly by chronic inflammatory back pain and radiographic sacroiliitis. Since genetic factors have been shown to be major determinants of susceptibility to the disease and contribute to amplification of immune responses, polymorphic variability in immune response genes are of interest. The interleukin-12p40 gene (IL12B) encodes the p40 polypeptide chain, which, together with $\mathrm{p} 19$, composes IL-23, a member of the IL-12 superfamily.

Objectives: In this study, we examined functional association between two $I L 12 B$ polymorphisms - IL12Bpro (rs17860508) and IL12B 3' UTRA/C (rs3212227), and cytokine production of both pro-inflammatory cytokines IL-12p40 and IL-23 in AS patients.

Methods: A total of 69 AS patients with a mean age $43 \pm 16$ years and 257 healthy individuals from Bulgarian population were genotyped. Genotyping for the rs3212227 was performed by restriction fragment length polymorphisms-PCR assay and for the rs 17860508 by allele specific-PCR. Serum IL-12p40 and IL-23 concentrations measurement was done using ELISA test.

Results: We found significant differences in the genotype $(p=0.029)$ and allele $(p=0.006)$ frequencies of $r s 17860508$ polymorphism between AS patients and controls. An association between AS and the rs17860508 polymorphism was established under the allelic model (allele 2 vs. allele $1 ; \mathrm{OR}=1.698$ ), the dominant model $(1.2+2.2$ vs. 1.1 ; OR=2.427), and the co-dominant model (2.2 vs. 1.1 ; 
$\mathrm{OR}=2.812 ; 1.2$ vs. 1.1; $\mathrm{OR}=2.252$ ). Genotype and allele frequencies of rs3212227 polymorphism of the IL12B were similar in cases and controls. The mean $( \pm$ SEM) levels of IL-12p40 and IL-23 in AS patients $(176.5 \pm 20.7 \mathrm{pg} / \mathrm{ml}$; $26.98 \pm 3.4 \mathrm{pg} / \mathrm{ml}$, respectively) was significantly higher than in controls $(88.8$ $\pm 9.6 \mathrm{pg} / \mathrm{ml} ; 12.1 \pm 1.5$; respectively) $(\mathrm{p}<0.001)$. Stratification based on IL12Bpro revealed significantly higher serum levels of IL-12p40 in AS patients with IL12Bpro 1.1 and 1.2 genotypes than controls. Highest IL-23 serum levels were associated with IL12Bpro 2.2 genotype. With regard of polymorphic variability of IL12B 3' UTR, AC genotype in AS has been linked to a higher IL-12p40 production and lower one of IL-23.

Conclusions: Allelic variants in the $I L 12 B$ genes have the potential to alter the expression of both IL-12p40 and IL-23 cytokines in specific manner for AS patients. Carriage of the IL12Bpro variant 1-allele and of the $3^{\prime}$-UTR A-allele in the genotype regardless of homo- or heterozygosity could enhance systemic IL$12 \mathrm{p} 40$ and IL-23 levels.

Disclosure of Interest: None declared

DOI: 10.1136/annrheumdis-2018-eular.3717

\section{SAT0021 INNATE LYMPHOID CELLS ARE NOT A MAIN SOURCE OF IL-17A IN THE INFLAMED SPONDYLOARTHRITIS JOINT}

N. Yeremenko ${ }^{1,2,3}$, I.C.J. Blijdorp ${ }^{1,2,3}$, L.J.J. Van Mens ${ }^{1,2}$, S. Menegatti ${ }^{4}$ S. Chen ${ }^{1,2,3}$, H.S. Hreggvidsdottir ${ }^{1,2,3}$, T. Noordenbos ${ }^{1,2}$, T.E. Latuhihin ${ }^{1,2,3}, \mathrm{~J}$. H. Bernink ${ }^{3,5}$, L. Rogge ${ }^{4}$, H. Spits ${ }^{3,5}$, D.L.P. Baeten ${ }^{1,2,3} \cdot{ }^{1}$ Department of Clinical Immunology and Rheumatology, ${ }^{2}$ Amsterdam Rheumatology and immunology Center, ${ }^{3}$ Laboratory of Experimental Immunology, Academic Medical Center, Amsterdam, Netherlands; ${ }^{4}$ Immunology, Institut Pasteur, Paris, France;

${ }^{5}$ Department of Cell Biology and Histology, Academic Medical Center, Amsterdam, Netherlands

Background: Clinical trials of the anti-IL-17A antibody secukinumab demonstrated the crucial role of IL-17A cytokine in the pathogenesis of spondyloarthritis (SpA), however, its cellular source in this condition remains controversial. Group 3 innate lymphoid cells (ILC3s) have been recently identified in a number of different tissues as potent producers of proinflamatory cytokines, including IL-17A and IL22

Objectives: In this study we set out to characterise the presence and composition of ILCs and investigate whether these cells are the important source of IL-17A in the synovial tissue of patients with SpA

Methods: Matched synovial tissue (ST), synovial fluid (SF) and peripheral blood (PB) were obtained from SpA and rheumatoid arthritis (RA) patients with actively inflamed knee joints. ILCs subsets were characterised by flow cytometry. Gene expression analysis at the single-cell level was performed directly ex vivo and after stimulation with PMA ionomycin. IL-17A ELISPOT assay was used to detect IL-17A-secreting cells.

Results: Analysis revealed that ILCs, and particularly NKp44-positive ILC3s, are expanded in the inflamed arthritic joint. Single cell expression analysis revealed that ST ILCs are clearly distinguishable from ST T cells and from their PB counterparts. We detected expression of Th17 signature transcripts RORC, AHR and IL$23 R$ in the notable fraction of ST ILC3s. Furthermore these cells were capable to induce IL-22, but not IL-17A expression in response to. in vitro re-stimulation Conclusions: We demonstrate in this study that ILC3s are absolutely and relatively enriched in the synovial joint of patients with SpA, however these cells are not a significant source of IL-17A cytokine in this pathology.

Disclosure of Interest: None declared

DOI: 10.1136/annrheumdis-2018-eular.6847

\section{SAT0022 MIR-15A/16 SUPPRESS INFLAMMATORY RESPONSE AND CELL INVASION AND MIGRATION IN FIBROBLAST- LIKE SYNOVIOCYTES IN RHEUMATOID ARTHRITIS VIA TARGETING SOX5}

W. Tan, Q. WU, A. Luo, Y. Wei, F. Wang, M. Zhang. Rheumatology, The First Affiliated Hospital of Nanjing Medical University, Nanjing, China

Background: Aberrant expressions of miRNAs play a critical role in the inflammatory and immune response in rheumatoid arthritis (RA). The microRNA cluster which encodes for miR-15a and miR-16, located at 13q14.3. Both these microRNAs have been implicated in the pathogenesis of many diseases, but little is known of their role in $\mathrm{RA}$.

Objectives: In this study, we aim to investigate the underlying molecular mechanisms of MiR-15a/16 in RA, which will provide new insight into understanding the pathogenesis of RA and identifying novel therapeutics targets for this disease.

Methods: The expression of MiR-15a/16 in the peripheral blood mononuclear cell (PBMC) and synovium from RA, osteoarthritis $(\mathrm{OA})$ and healthy controls $(\mathrm{HC})$ was determined by RT-qPCR and Western blotting. Mimics of MiR-15a/16 were transfected into the human rheumatoid fibroblast-like synoviocytes (FLS) MH7A cell line. The effect of MiR-15a/16 on proinflammatory cytokines expression, migration and invasion of FLS was detected by RT-qPCR, transwell and F-actin staining. The potentially target gene of miR-15a/16 was predicted by bioinformatics analysis. The 3'UTR of Sox 5 containing wild-type or mutated miR-15a/16 binding sites was cloned to the downstream of a luciferase vector and transfected into MH7A, respectively

Results: There is no significant difference of MiR-15a/16 expression in PBMC in $\mathrm{RA}$ and $\mathrm{HC}$ at baseline. However, the MiR-15a/16 expression is lower in PBMC from non-responders (defined DAS28 changes $<1.2$ after 3 months DMARDs therapy) than those in responders after 3 months DMARDs therapy. The levels of MiR-15a/16 expression were significant decreased in the synovium from RA patients, as compared with OA patients. Transfection of MH7A with MiR-15a and 16 mimic suppressed IL-1b, TNFa, IL-17 expression, decreased cell migration and invasion and affected the cytoskeletal reorganisation in RA-FLS. We cotransfected MH7A with luciferase-reporter constructs with either wild-type or mutated miR-132 or miR-15a/16 mimics. Luciferase assay showed that either miR-15a or 16 reduced the luciferase intensity of Sox $53^{\prime}$ UTR. However, mutated miR-15a/16 alleviated the inhibitory effect of miR-15a/16 on the intensity of Sox 5 3'UTR. To determine whether Sox 5 mediates the roles of miR-15a/16 in cell migration and invasion, we constructed recombinant Adenovirus SOX5 recombinant Adenovirus SOX5 overexpressing vector. We then found that overexpression of Sox 5 restored the cell invasion, and migration in $\mathrm{MH} 7 \mathrm{~A}$ that were inhibited by and miR-15a/16.

Conclusions: In conclusion, our data indicate that MiR-15a/16 play an important role in inflammatory response and cell invasion and migration in RA-FLS by directly targeting Sox5, and imply that these miRNAs have potential as therapeutic targets for RA.

Disclosure of Interest: None declared

DOI: 10.1136/annrheumdis-2018-eular.4151

\section{SAT0023 CONTROL OF CYTOKINE MRNA DEGRADATION BY THE HISTONE DEACETYLASE INHIBITOR ITF2357 IN RHEUMATOID ARTHRITIS FIBROBLAST-LIKE SYNOVIOCYTES}

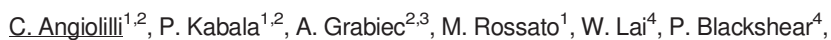
K. Reedquist ${ }^{1,2}$, D. Baeten ${ }^{2}$, T. Radstake ${ }^{1} .{ }^{1}$ Department of Rheumatology and Clinical Immunology, Laboratory of Translational Immunology, University Medical Center Utrecht, Utrecht, ${ }^{2}$ Department of Clinical Immunology and Rheumatology and Department of Experimental Immunology, Amsterdam Rheumatology and Immunology Center, Amsterdam, Netherlands; ${ }^{3}$ Department of Microbiology, Faculty of Biochemistry, Biophysics and Biotechnology, Jagiellonian University, Kraków, Poland; ${ }^{4}$ Signal Transduction Laboratory, National Institute of Environmental Health Sciences, Research Triangle Park, NC, USA

Background: Control of cytokine mRNA degradation acts as an essential check point to limit the overproduction of inflammatory proteins. ${ }^{1}$ In rheumatoid arthritis (RA), altered expression of the mRNA-degrading protein TTP (tristetraprolin, ZFP36) has been recently reported in synovial tissue, possibly contributing to the perpetuating inflammatory loop in the synovium. ${ }^{2}$ Histone deacetylase inhibitors (HDACi) are small molecule drugs suppressing cytokine production in vitro and in vivo and displaying initial safety and efficacy in the treatment of systemic onset juvenile idiopathic arthritis. ${ }^{3,4}$ However, their transcriptional and post-transcriptional mechanisms of action are not yet completely characterised.

Objectives: We aimed to investigate the mRNA degrading properties of the HDACi ITF2357 on a panel of inflammatory mediators in RA fibroblast-like synoviocytes (FLS).

Methods: The effects of ITF2357 on the expression and mRNA stability of IL-1 $\beta$ inducible genes in FLS were analysed using array-based qPCRs and Luminex. The expression of primary and mature cytokines transcripts, the mRNA levels of TTP and other AU-rich element binding proteins (ARE-BP) and the cytokine profile of fibroblasts derived from $Z F P 36^{+/+}$and $\mathrm{ZFP}^{-/-}$mice was measured by qPCR. ARE-BP silencing was performed by siRNA-mediated knockdown, and TTP post-translational modifications analysed by immunoblotting.

Results: ITF2357 reduced the expression of $85 \%$ of the analysed IL-1 $\beta$-inducible transcripts, including cytokines (IL6, IL8), chemokines (CXCL2, CXCL5, CXCL6, CXCL10), matrix-degrading enzymes (MMP1, ADAMTS1) and other inflammatory mediators. Analyses of mRNA stability demonstrated that ITF2357 accelerates IL6, IL8, PTGS2, and CXCL2 mRNA degradation, a phenomenon associated with the enhanced transcription of TTP, but not other ARE-BP, and the altered posttranslational status of TTP protein. TTP knockdown potentiated cytokine production in RA FLS and murine fibroblasts.

Conclusions: Our study identifies that regulation of cytokine mRNA stability is a predominant mechanism underlying ITF2357 anti-inflammatory properties, occurring via regulation of TTP. These results highlight the therapeutic potential of ITF2357 in the treatment of RA. 